Kinetoplastid membrane protein 11 induces pores in anionic phospholipid membranes: Effect of cholesterol

Animesh Halder ${ }^{1 \#}$, Achinta Sannigrahi ${ }^{2 \#}$, Nayan $\mathrm{De}^{2}$, Krishnananda Chattopadhyay ${ }^{\dagger 2}$ and Sanat Karmakar $^{1 \dagger}$

${ }^{1}$ Soft matter and Biophysics Laboratory, Department of Physics, Jadavpur University, 188, Raja S. C. Mallick Road, Kolkata 700032, India

${ }^{2}$ Structural Biology \& Bio-Informatics Division, CSIR-Indian Institute of Chemical Biology, 4, Raja S. C. Mallick Road, Kolkata 700032, India

\# contributed equally

†Corresponding author: emails: sanat.karmakar@jadavpuruniversity.in, krish@,iicb.res.in

\title{
Antimicrobial Index:
}

We used AMPA (http://tcoffee.crg.cat/apps/ampa), an automated web server for prediction of KMP-11 antimicrobial regions. The AMPA algorithm is established using a high throughput screening results from AMP beactenecin 2A on the basis of an antimicrobial propensity scale. From these data, an antimicrobial index (AI) can be evaluated depending on fair estimation of the propensity for amino acids to be present in an AMP sequence. It has been suggested that amino acids with low antimicrobial index are the most favored to be part of antimicrobial peptides. The antimicrobial profile has been drawn using sliding window method. This method suggested that the regions of a protein located below the threshold value are considered as antimicrobial domain. Here, using AMPA, we calculated the antimicrobial region of KMP-11. We used the sequence of a well known antimicrobial peptide, magainin as a positive control. 


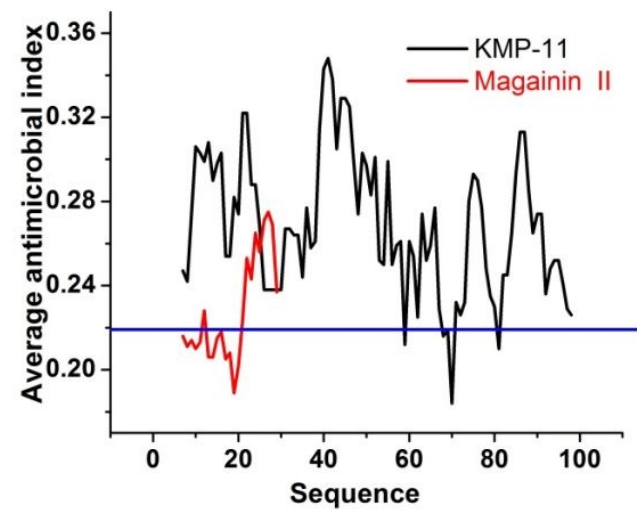

Figure S1: Average antimicrobial index against the sequence stretch of WT KMP-11 and magainin-II. The blue line suggests the threshold value of average antimicrobial index.

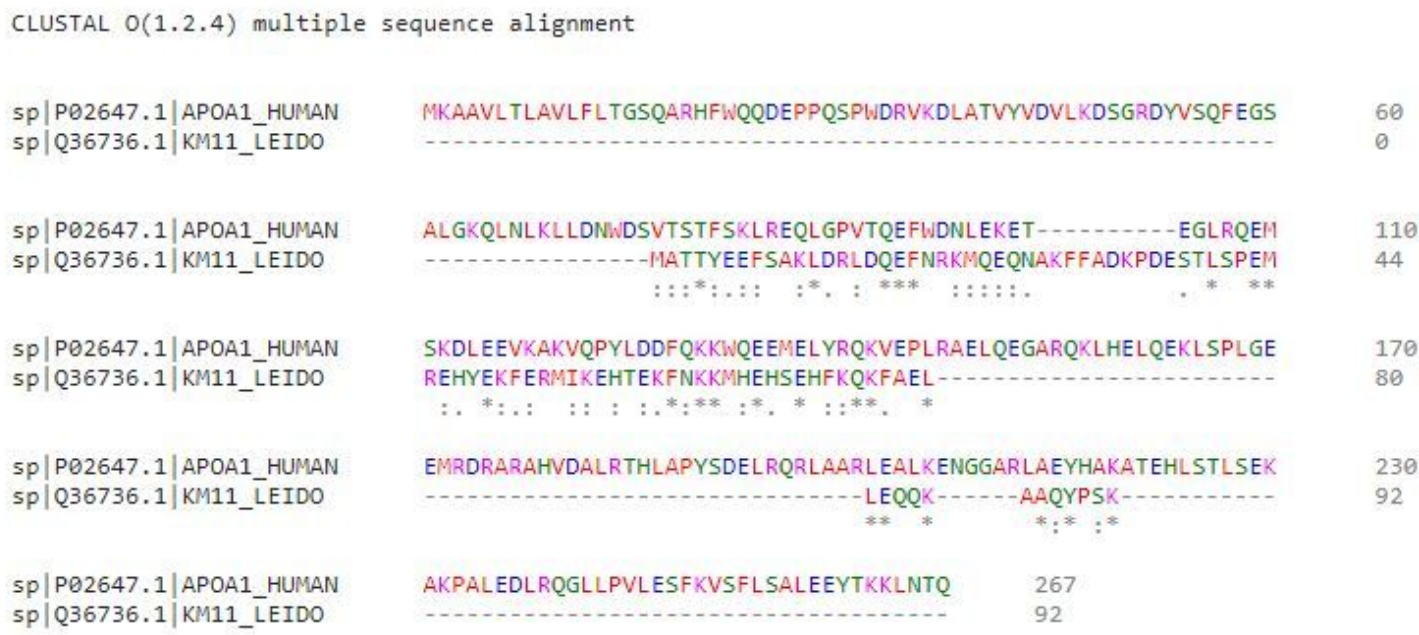

Figure S2: The sequence similarity between KMP-11 and APOA-1 protein as determined from the clustal omega derived multiple sequence alignment. Alignment showed $27 \%$ sequence similarity 


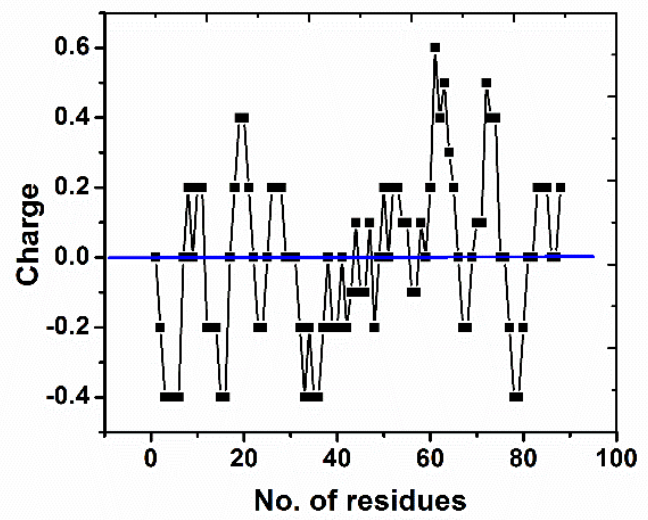

Figure S3: Charge distribution of the amino acid residues of KMP-11 throughout the entire sequence of protein calculated using EMBOSS software. 


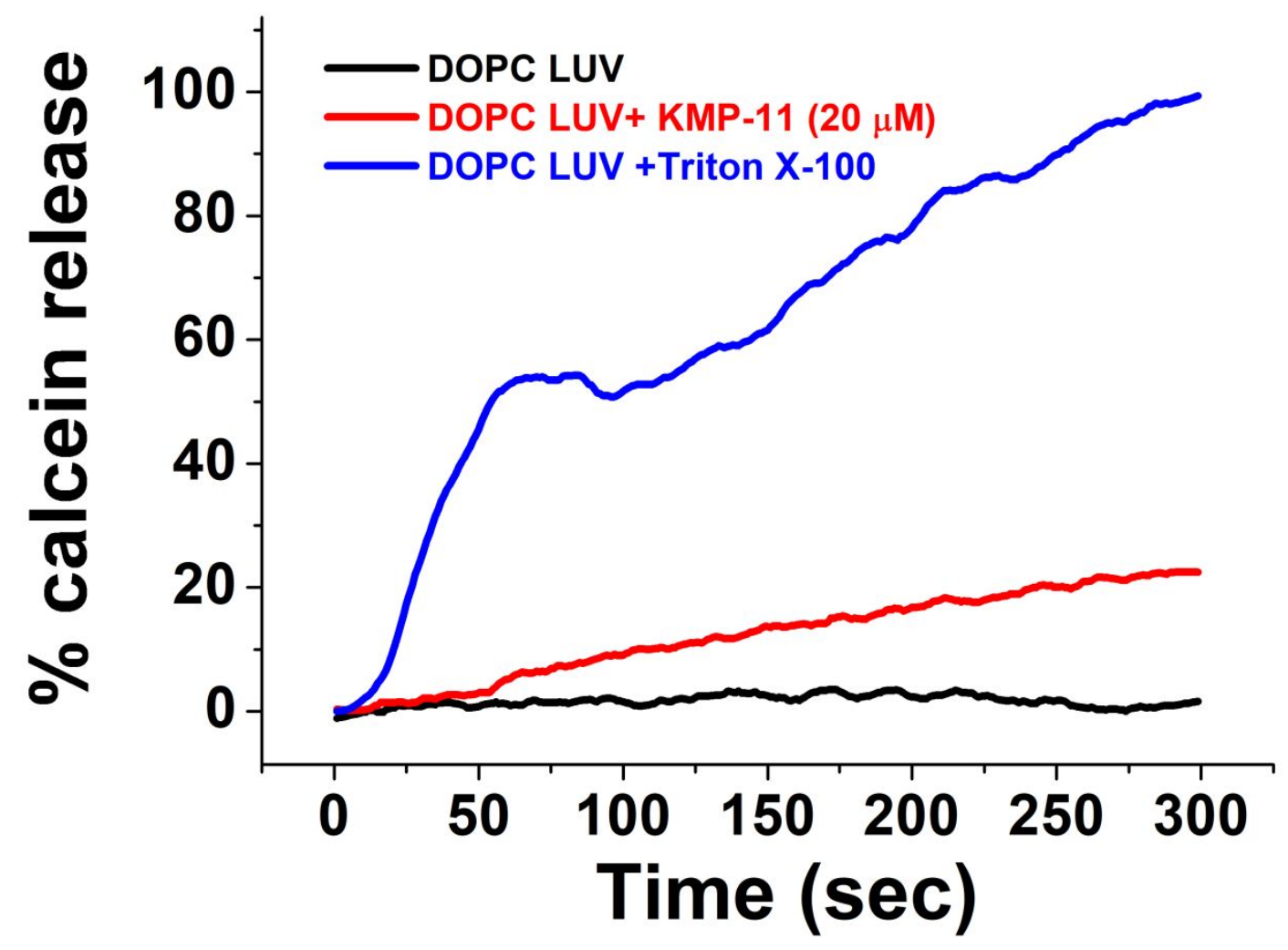

Figure S4: Dye leakage spectra of calcein entrapped LUVs composed of DOPC lipid in presence of KMP11 (concentration was $20 \mu \mathrm{M}$ ) and in presence of triton X-100. 


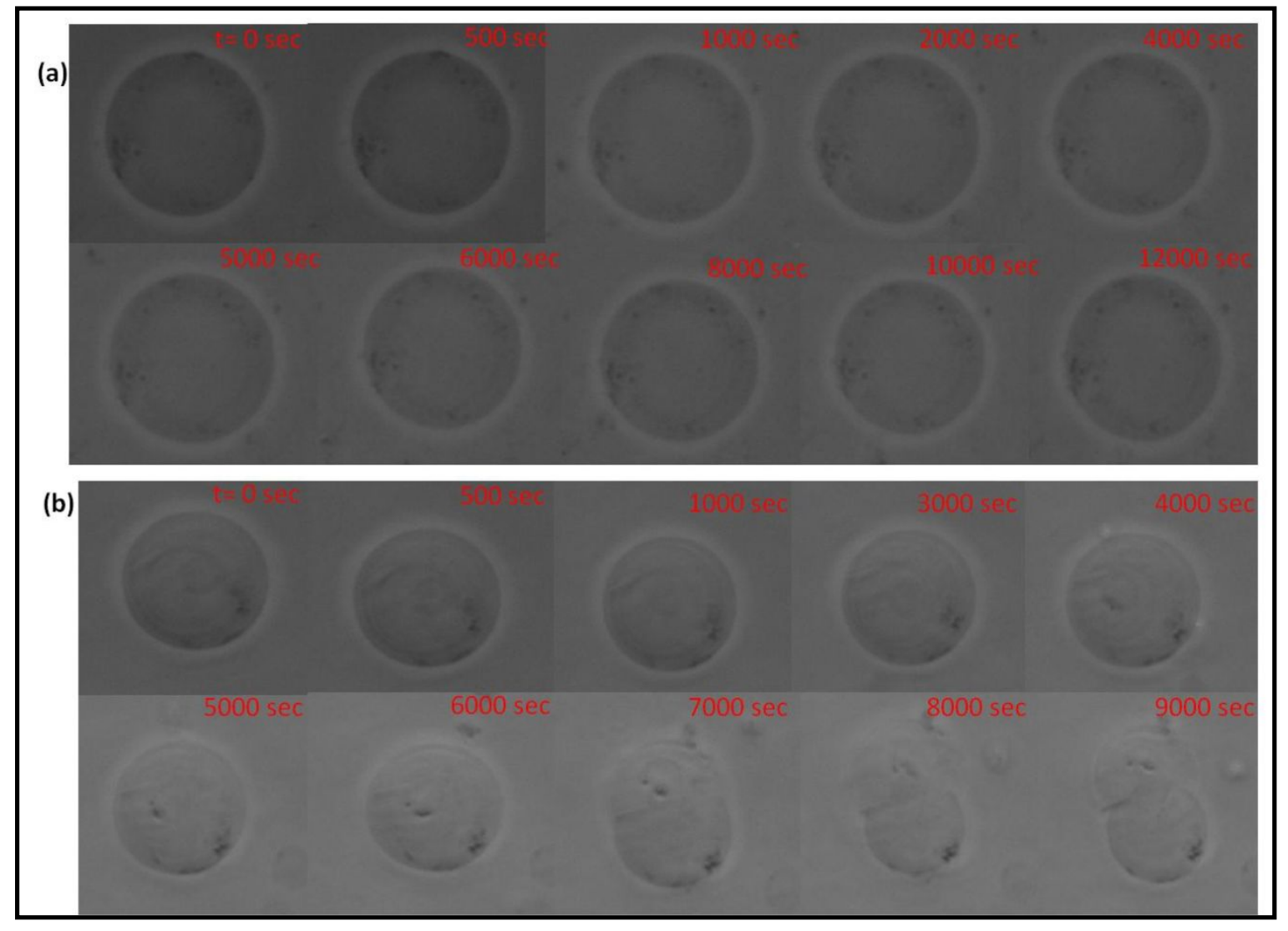

Figure S5: Phase contrast micrographs of GUV composed of DOPC-DOPG (4:1) exposed to (a) $5 \mu \mathrm{M}$ and (b) $10 \mu \mathrm{M}$ concentrations of $\mathrm{KMP}-11$. The size of the vesicle $\sim 34 \mu \mathrm{m}$ 


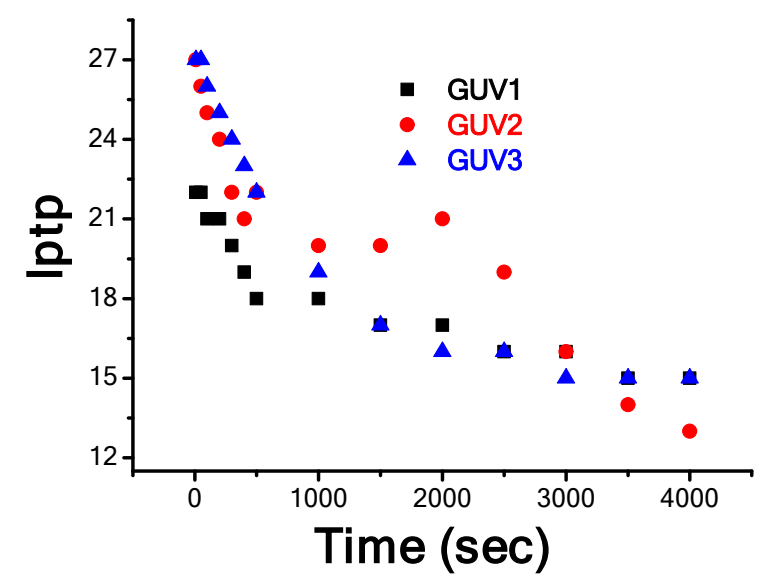

Figure S6: Change in $\mathrm{I}_{\mathrm{ptp}}$ values of three different GUVs when these were treated with $20 \mu \mathrm{M}$ concentration of KMP-11. 


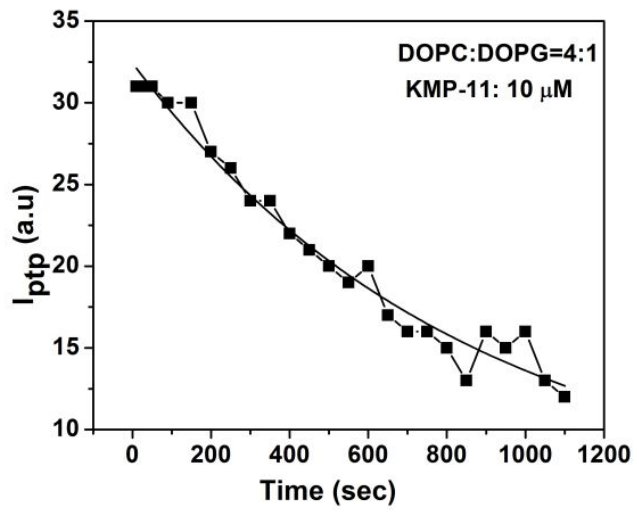

Figure S7: The decay of $I_{p t p}$ (peak to peak intensity) with time for GUV composed of DOPC-DOPG. Solid line represents the fit curve obtained from single exponential decay and decay rate constant $(\tau)$ was found to be $0.0012 \mathrm{sec}^{-1}$. 